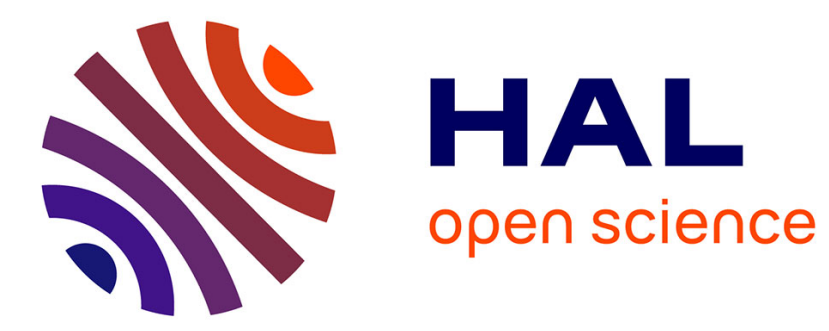

\title{
Exploiting Map Information for Driver Intention Estimation at Road Intersections
}

Stéphanie Lefèvre, Christian Laugier, Javier Ibañez-Guzmán

\section{To cite this version:}

Stéphanie Lefèvre, Christian Laugier, Javier Ibañez-Guzmán. Exploiting Map Information for Driver Intention Estimation at Road Intersections. 2011 IEEE Intelligent Vehicle Symposium, Jun 2011, Baden-Baden, Germany. pp.583-588. inria-00588743

\section{HAL Id: inria-00588743 https://hal.inria.fr/inria-00588743}

Submitted on 26 Apr 2011

HAL is a multi-disciplinary open access archive for the deposit and dissemination of scientific research documents, whether they are published or not. The documents may come from teaching and research institutions in France or abroad, or from public or private research centers.
L'archive ouverte pluridisciplinaire $\mathbf{H A L}$, est destinée au dépôt et à la diffusion de documents scientifiques de niveau recherche, publiés ou non, émanant des établissements d'enseignement et de recherche français ou étrangers, des laboratoires publics ou privés. 


\title{
Exploiting Map Information for Driver Intention Estimation at Road Intersections
}

\author{
Stéphanie Lefèvre, Christian Laugier, Javier Ibañez-Guzmán
}

\begin{abstract}
Safety applications at road intersections require algorithms that can estimate the manoeuvre intention of all the drivers in the scene. In this paper, the use of contextual information extracted from a digital map of the road network is explored. We propose a Bayesian network which combines probabilistically uncertain observations on the vehicle's behaviour and information about the geometrical and topological characteristics of the road intersection in order to infer a driver's manoeuvre intention. The approach is evaluated on trajectories recorded from real traffic, including complex scenarios where the behaviour of the vehicle is inconsistent. We define an evaluation method that accounts for the impossibility to make reliable predictions in some situations, and show that the system is able to reliably combine vehicle state information and map information to infer a driver's intended manoeuvre.
\end{abstract}

\section{INTRODUCTION}

The use of passive safety systems on board passenger vehicles has had a significant impact on reducing the number of road accident fatalities in the past 30 years. According to a study conducted in three European countries between 1980 and 2000 [1] these systems are responsible for a $30 \%$ to $40 \%$ decrease in the number of road accident fatalities over that period. However since the mid-90's the number of people killed in road accidents has not decreased significantly, and the trend is levelling-off [2]. While passive safety systems are activated once an accident occurs, a further reduction in fatalities will be achieved by systems that avoid or mitigate accidents. These are known as active safety systems and form part of the Advanced Driver Assistance Systems (ADAS) found in modern vehicles.

Road intersections represent the most complex configurations found in a road network and are the locations of many accidents. In Europe, the U.S. and Japan between $40 \%$ and $60 \%$ of road accidents resulting in injuries occur at intersections. Recent studies identified driver error as the main cause, in particular inattention and misinterpretation of the situation [3], [4]. Therefore, if the driver can be warned in a relevant manner while he is approaching and/or traversing an intersection, a major contribution towards the reduction of accidents could be made.

In order to provide relevant information to the driver, a system must be capable of understanding what is currently happening at the intersection. This is known in the literature as

S. Lefèvre and C. Laugier are with INRIA Grenoble Rhône-Alpes, 655 av. de l'Europe - Montbonnot, 38334 Saint Ismier Cedex, France, \{stephanie.lefevre, christian.laugier\}@inrialpes.fr

S. Lefèvre and J. Ibañez-Guzmán are with Renault S.A., 1 av. du Golf, 78288 Guyancourt, France, \{stephanie.lefevre, javier.ibanezguzman\}@renault.com the situation assessment problem [5]. Algorithms are needed that can estimate the current manoeuvre intention of all the drivers in the scene. This is a crucial task since knowing the intention of the drivers is needed to predict the evolution of the scene and therefore to identify potential collisions. The work presented here was conducted while visiting the Stanford Driving Group using their autonomous vehicle Junior 2. The robot was built based on Junior, the Stanford entry in the DARPA Urban Challenge [6]. Although the robot was able to successfully negotiate intersections during the competition [7], the need to improve situation assessment at road intersections was identified.

Estimating the manoeuvre intention of a driver at a road intersection is a challenging task. The difficulty lies in the high number of possible scenarios that the system must be able to interpret. In this paper we claim that the contextual information contained in the map of the road network (i.e. the spatial description of the road intersection) gives a useful indication of what a driver intends to do. We also claim that handling uncertainties on the input information is necessary to make reliable predictions. In Sec. II we review related works and formulate the problem addressed. Sec. III describes the Bayesian network that we propose as a solution. The performance is evaluated using data recorded from real traffic in Sec. IV, both when the driver's behaviour is consistent and when it is inconsistent.

\section{SituAtion ASSESSMENT AT ROAD INTERSECTIONS}

\section{A. Related works}

Approaches for situation assessment at intersections can be classified in two main categories: discriminative approaches and generative approaches.

Discriminative approaches classify driver intention without modelling explicitly the underlying distribution of variables. A popular approach is to learn trajectory prototypes for each intersection targeted by the application, using trajectories that have been observed at that specific intersection in the past. It is then possible to predict the future manoeuvre of a vehicle by matching its trajectory with the prototype trajectories. This solution was adopted by [8] and extended by [9] in order to take into account interactions between vehicles. The main drawback of this method is that the prototype trajectories learned at the training intersections cannot be reliably generalised to arbitrary intersections. The many possible variations of intersection layouts, combined with the large number of possible scenarios at an intersection, make it very challenging 
in practice to obtain enough training data and to define input features that are generic to all the possible scenarios.

Generative methods explicitly model the process between driver intention and vehicle behaviour. In [10] the basic elements constituting manoeuvres are recognised using fuzzy rules combined with a Bayesian filter, and a Probabilistic Final State Machine (PFSM) is used to represent the possible sequences of basic elements of several driving manoeuvres. The approach proposed in [11] is to model driver intention at an intersection using a PFSM where the transition probabilities are set dynamically using the output of a continuous vehicle state tracker. These works do not take into account contextual information; they infer driver intention from vehicle state only. The approach described in [12] uses Hierarchical Hidden Markov Models (HHMMs) to estimate manoeuvre intention. The possible evolution of the vehicle motion for a given manoeuvre is represented in a probabilistic manner by Gaussian Processes (GPs). This representation makes it possible to integrate information about the road geometry as well as uncertainties on the realisation of a manoeuvre. The European PReVENT-INTERSAFE project [13] integrated knowledge about the topology of an intersection to perform probabilistic driver intention estimation, but did not take into account knowledge about the geometry of the intersection.

\section{B. Problem addressed}

Generally in the literature of manoeuvre intention estimation the information on the layout of the intersection is not used. In the rare cases where it is, the topology and the geometry are not considered together and lane-level positioning is assumed so that hard lane assignment can be performed. In this paper we focus on these two aspects of manoeuvre intention estimation:

1) Incorporating knowledge about the layout of the intersection: this is necessary in order for a system to be generic to arbitrary intersections.

2) Taking into account the uncertainties on the input information: in order to perform hard lane assignment it was shown [13] that the required accuracy of the digital map and vehicle positioning is $0.3 \mathrm{~m}$, which is currently not realistic. Therefore it is necessary to account for uncertainties while determining the lane on which a vehicle is driving.

The problem addressed in this paper is to estimate - for all the relevant vehicles in the scene - the driver's Intended manoeuvre from observations on the Vehicle behaviour and knowledge about the Context of the situation.

- Intended manoeuvre: the lane through which the driver intends to exit the intersection.

- Context: the layout of the intersection, i.e. topological and geometrical characteristics of the intersection.

- Vehicle behaviour: succession of vehicle positions, orientations, and turn signals.

In this work we do not use the clues provided by the kinematic behaviour of the vehicle (speed and acceleration) to estimate a

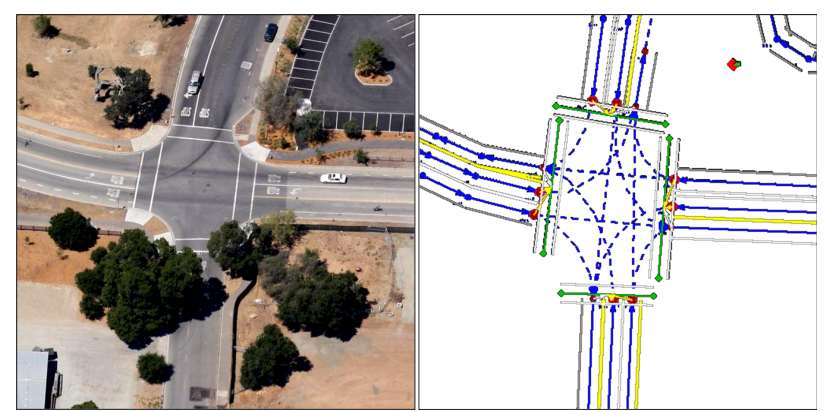

Figure 1. This intersection (satellite imagery on the left, RNDF representation on the right) has 4 roads, 8 entrance lanes, 4 exit lanes and 12 crossing connections. The blue arrows represent the centre of lanes and the dotted arrows inside the intersection area are the exemplar paths generated for each crossing connection.

driver's intention. Our decision to discard them was motivated by the fact that the kinematic behaviour of a vehicle at an intersection is mainly a consequence of the traffic rules and of the presence of other vehicles, therefore it does not make sense to interpret the speed and acceleration of a vehicle without taking into account interactions between vehicles. In ongoing work [14] we take into account traffic rules and the presence of other vehicles to interpret the pose and kinematic behaviour of a vehicle and estimate both the manoeuvre intention and yielding intention of the driver.

\section{CONTEXT-BASEd BAyESiAn NETWORK}

As a solution to the problem defined in Sec. II-B we propose a Bayesian Network $(\mathrm{BN})$ with uncertain evidence.

\section{A. Extraction of contextual information from a digital map}

The digital map of the road network used in this work is defined in the Route Network Definition File (RNDF) format, a standard created for the DARPA Urban Challenge [6]. The RNDF representation of an intersection consists of topological and geometrical information: a list of roads, lanes, and authorised crossing connections. In addition to this information, an exemplar path is generated for each crossing connection. An exemplar path is a generic path that a vehicle can take inside the intersection. This representation is illustrated in Fig. 1. A tool was developed to generate a map in the RNDF format from manually annotated satellite images; the resulting map has an accuracy of approximately $0.5 \mathrm{~m}$. From this representation we extract topological and geometrical information that is later used to infer driver intention at the intersection.

1) Variables extracted directly from the digital map:

$\left\{r_{i}\right\}_{1}^{N_{R}}$ are the $N_{R}$ roads of the intersection

$\left\{l_{i}\right\}_{1}^{N_{L}}$ are the $N_{L}$ entrance lanes of the intersection

$\left\{m_{i}\right\}_{1}^{N_{M}}$ are the $N_{M}$ exit lanes of the intersection

$\left\{p_{i}\right\}_{1}^{N_{P}}$ are the $N_{P}$ exemplar paths of the intersection

\section{2) Functions that extract the topological characteristics:}

$\operatorname{bel}\left(l_{i}, r_{i}\right)$ is true if entrance lane $l_{i}$ belongs to road $r_{i}$

$\operatorname{bel}\left(m_{i}, r_{i}\right)$ is true if exit lane $m_{i}$ belongs to road $r_{i}$

leads $\left(l_{i}, m_{i}\right)$ is true if exit lane $m_{i}$ can be reached from entrance lane $l_{i}$

leads $\left(l_{i}, p_{i}\right)$ is true if path $p_{i}$ can be reached from entrance lane $l_{i}$ 


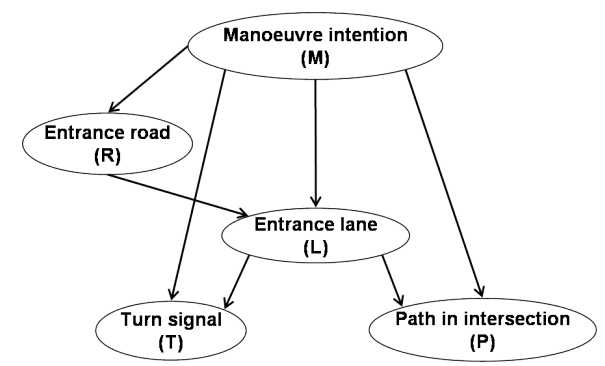

Figure 2. Graphical model corresponding to the decomposition of Eq. 1.

leads $\left(p_{i}, m_{i}\right)$ is true if exit lane $m_{i}$ can be reached from path $p_{i}$ $\operatorname{most}\left(l_{i}, m_{i}\right)$ is true if exit lane $m_{i}$ is the leftmost or rightmost exit lane that can be reached from entrance lane $l_{i}$

one $\left(l_{i}\right)$ is true if only one exit lane can be reached from entrance lane $l_{i}$ side $\left(l_{i}, l_{j}\right)$ returns right (resp. le ft) if $l_{j}$ is located to the right (resp. left) of $l_{i}$, with entrance lanes $l_{i}$ and $l_{j}$ belonging to the same road

$\operatorname{count}\left(r_{i}, \mathcal{C}_{i}\right)$ returns the number of roads which comply with condition $\mathcal{C}$ $\operatorname{count}\left(l_{i}, \mathcal{C}_{i}\right)$ returns the number of lanes which comply with condition $\mathcal{C}_{i}$

\section{3) Functions that extract the geometrical characteristics:}

$v_{t}$ is defined as the position of a vehicle at time $t$ $\operatorname{dist}\left(v_{t}, m_{i}\right)$ returns the distance between $v_{t}$ and exit lane $m_{i}$

$\operatorname{dist}\left(l_{i}, m_{i}\right)$ returns the distance between entrance lane $l_{i}$ and exit lane $m_{i}$ angle $\left(l_{i}, m_{i}\right)$ returns the angle between entrance lane $l_{i}$ and exit lane $m_{i}$ angle $\left(v_{t}, p_{i}, m_{i}\right)$ returns the angle between the tangent to path $p_{i}$ at point $v_{t}^{\prime}$ and exit lane $m_{i}$, where $v_{t}^{\prime}$ is the projection of $v_{t}$ on path $p_{i}$

\section{B. Bayesian network model}

1) Variables definition:

- $R \in\left\{r_{i}\right\}_{1}^{N_{R}}$ : the road through which the vehicle reaches the intersection.

- $L \in\left\{l_{i}\right\}_{1}^{N_{L}}$ : the entrance lane through which the vehicle reaches the intersection.

- $T \in\{$ left,right, none $\}$ : the turn signal that is on.

- $P \in\left\{p_{i}\right\}_{1}^{N_{P}}$ : the exemplar path that the vehicle follows in the intersection.

- $M \in\left\{m_{i}\right\}_{1}^{N_{M}}$ : the exit lane through which the vehicle leaves the intersection ( $\mathrm{M}=$ manoeuvre intention).

2) Decomposition: The decomposition is defined in Eq. 1 and illustrated by the graphical model in Fig. 2.

$$
\begin{aligned}
P(M, R, T, L, P)= & P(M) \times P(R \mid M) \times P(T \mid L, M) \\
& \times P(L \mid R, M) \times P(P \mid L, M)
\end{aligned}
$$

3) Specification of the conditional probabilities: The conditional probabilities of Eq. 1 encode our knowledge on how a vehicle's behaviour is influenced by the intended manoeuvre and the geometry and topology of the intersection. $P\left(m_{i}\right)$ is set to uniform because we have no prior knowledge about which manoeuvres are more likely to be performed. The other conditional probabilities are set using a rule-based algorithm that uses the contextual information extracted from the digital map (see Sec. III-A). The pseudocode for the algorithm is given below.

- $P\left(r_{i} \mid m_{i}\right)$

$\mathcal{C}_{1}=\exists l_{i}: \operatorname{bel}\left(l_{i}, r_{j}\right) \& \operatorname{leads}\left(l_{i}, m_{j}\right) \& \operatorname{bel}\left(m_{i}, r_{k}\right) \& \operatorname{bel}\left(m_{j}, r_{k}\right)$

IF $\exists l_{i}: \operatorname{bel}\left(l_{i}, r_{i}\right)$ \& leads $\left(l_{i}, m_{i}\right)$

THEN $P\left(r_{i} \mid m_{i}\right)=(1-\alpha) \times 1 /\left(\operatorname{count}\left(r_{j}, \mathcal{C}_{1}\right)\right)$

ELSE $P\left(r_{i} \mid m_{i}\right)=\alpha \times 1 /\left(\operatorname{count}\left(r_{j}, \overline{\mathcal{C}_{1}}\right)\right)$
Only topological characteristics of the intersection are used. $\alpha$ is a constant variable that represents the probability that a driver performs a forbidden manoeuvre (in terms of allowed [entrance road, exit lane] pairs).

$$
\text { - } P\left(t_{i} \mid l_{i}, m_{i}\right)
$$

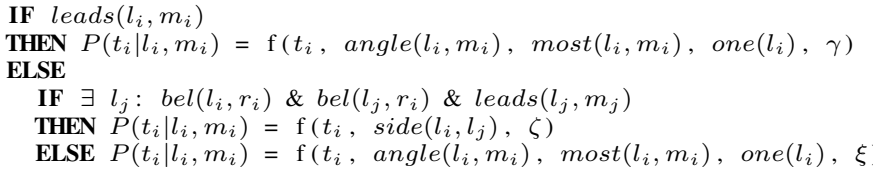

Topological and geometrical characteristics of the intersection are used. $\gamma, \zeta$ and $\xi$ are multivariate constant variables which represent the probability that a driver puts on a turn signal in various scenarios (e.g. the probability that a driver puts on a turn signal while performing a forbidden manoeuvre). We take into account that turn signals can also indicate an intention to change lanes.

$$
\text { - } P\left(l_{i} \mid r_{i}, m_{i}\right)
$$

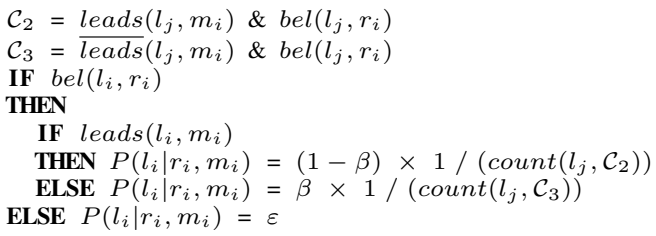

Only topological characteristics of the intersection are used. $\beta$ is a constant variable that represents the probability that the driver performs a forbidden manoeuvre (in terms of allowed [entrance road, entrance lane, exit lane] triplets).

$$
\text { - } P\left(p_{i} \mid l_{i}, m_{i}\right)
$$

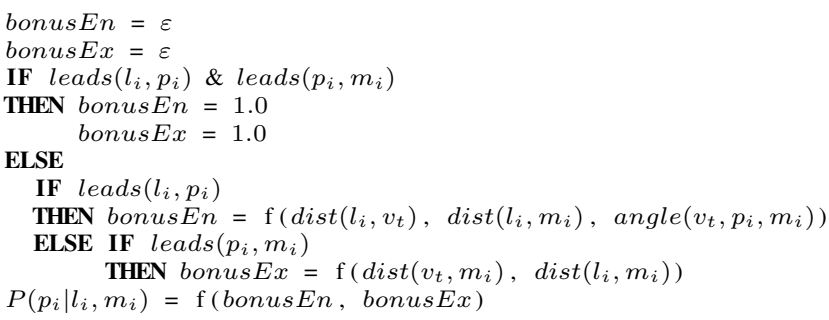

Topological and geometrical characteristics of the intersection are used. We use the current position of the vehicle as well as the distances and angles to various features of the intersection in order to decide which path inside the intersection would be the most appropriate to reach a specific exit lane from the vehicle's current pose.

$\alpha, \beta, \gamma, \zeta$ and $\xi$ are arbitrarily set but should be learned from data in the future.

\section{Inference with uncertain evidence}

The goal is to infer a probability distribution over $M$ from information on $L, T$ and $P$. In this work we consider that evidence on the values of $L$ and $P$ is uncertain, because the accuracy of the digital map and vehicle positioning is not high enough to perform hard lane and path assignment (see Sec. II-B). Instead we perform probabilistic lane and path assignment and integrate this uncertain evidence in the BN using Pearl's method of virtual evidence, as described below. 
1) Probabilistic lane and path assignment: Probabilistic lane assignment consists in generating a discrete probability distribution $\lambda_{L}=\left(\lambda_{l_{1}}, \ldots, \lambda_{l_{N_{L}}}\right)$, where $\lambda_{l_{i}} \propto P\left(L=l_{i}\right)$. We represent a lane as a bivariate normal distribution on successive poses (position, orientation), therefore $\lambda_{l_{i}}$ can be calculated as:

$$
\lambda_{l_{i}}=\exp \left(-\frac{1}{2}\left(\frac{\delta_{i}^{2}}{\sigma_{\delta}^{2}}+\frac{\theta_{i}^{2}}{\sigma_{\theta}^{2}}\right)\right)
$$

where $\delta_{i}$ is the distance between the vehicle's position and its orthogonal projection on lane $l_{i}, \theta_{i}$ is the angle between the vehicle's orientation and the orientation of lane $l_{i}, \sigma_{\delta}$ (resp. $\sigma_{\theta}$ ) is the standard deviation set for the distance (resp. the angle). In this work $\sigma_{\delta}$ and $\sigma_{\theta}$ are fixed, but a method to take into account the covariance matrices of the vehicle state and of the map can be found in [15].

The same approach is used to perform probabilistic path assignment, i.e. to calculate $\lambda_{P}=\left(\lambda_{p_{1}}, \ldots, \lambda_{p_{N_{P}}}\right)$.

2) Inference: Uncertain evidence in BNs has been defined as a generalisation of standard (hard) evidence in BNs by Pearl [16]. The idea is to recast uncertain evidence as hard evidence on some virtual event. Applying Pearl's theory to our BN, we add a virtual node $V_{L}$ as a child of $L$ and a virtual node $V_{P}$ as a child of $P$. $V_{L}$ and $V_{P}$ are binary variables always observed to be 1 . The conditional probabilities $P\left(V_{L} \mid L\right)$ and $P\left(V_{P} \mid P\right)$ incorporate the uncertain information $\lambda_{L}$ and $\lambda_{P}$ :

$$
\begin{cases}P\left(V_{L}=1 \mid L=l_{i}\right) & =\lambda_{l_{i}} \\ P\left(V_{P}=1 \mid P=p_{i}\right) & =\lambda_{p_{i}}\end{cases}
$$

The joint probability function of the $\mathrm{BN}$, previously defined by Eq. 1, therefore becomes:

$$
\begin{aligned}
P\left(M, R, T, L, P, V_{L}, V_{P}\right)= & P(M) \times P(R \mid M) \times P(T \mid L, M) \\
& \times P(L \mid R, M) \times P(P \mid L, M) \\
& \times P\left(V_{L} \mid L\right) \times P\left(V_{P} \mid P\right)
\end{aligned}
$$

Applying Pearl's method, the Bayesian question to infer $M$ from hard evidence on $T$ and uncertain evidence on $L$ and $P$ is $P\left(M \mid T, V_{L}, V_{P}\right)$, with $T$ the observed value of the turn signal and $V_{L}=V_{P}=1$. In this work the question is solved using exact inference.

\section{EXPERIMENTAL EVALUATION}

The system was tested on 42 vehicle trajectories recorded from real traffic at two four-way-stop intersections (including the intersection in Fig. 1). The trajectories (successive poses of the vehicles) were manually annotated on laser data. For each trajectory two sets of turn signal state annotations were automatically generated so that the system's performance could be evaluated on two types of scenarios:

- Consistent behaviour scenarios: the turn signal $T$ is consistent with the executed manoeuvre $M$ (e.g. no turn signal or left turn signal on for a left turn).

- Inconsistent behaviour scenarios: the turn signal $T$ is not consistent with the executed manoeuvre $M$ (e.g. right turn signal on for a left turn).

\section{A. Qualitative evaluation}

The objective is to evaluate the system's ability to make reliable interpretations of a vehicle's behaviour. Fig. 3 shows the output of the system for a left turn manoeuvre scenario. In this example the allowed manoeuvres for a vehicle approaching the intersection from the south are: turn left / go straight from the left lane and turn right / go straight from the right lane. The results can be interpreted as follows:

- Consistent behaviour scenario: the vehicle reaches the intersection through the left lane with the left turn signal on $(1 \mathrm{a}, 1 \mathrm{~b})$. The system interprets this as a high probability that the driver's intention is to turn left, and a much smaller probability that the driver's intention is to go straight. The system becomes increasingly confident that the driver's intention is to turn left as the vehicle makes its way through the intersection and is obviously aiming at a specific exit lane (1c, 1d).

- Inconsistent behaviour scenario: the vehicle reaches the intersection through the left lane with the right turn signal on $(2 \mathrm{a}, 2 \mathrm{~b})$. On $2 \mathrm{a}$ the vehicle is still far away from the intersection so this behaviour is interpreted as an intention to change lanes. There is also a chance that the vehicle is actually on the right lane (i.e. positioning error), which explains why the probability of turning right is higher than the probability of going straight. When the vehicle gets closer to the intersection (2b) the system has no preferred interpretation because this behaviour makes little sense. A human would come to the same conclusion and consider all manoeuvres to be equally likely. As the vehicle executes the manoeuvre inside the intersection it becomes more and more obvious that the vehicle will turn left since the other manoeuvres are no longer feasible, therefore the uncertainty of the system's estimation decreases $(2 \mathrm{c}, 2 \mathrm{~d})$.

The system was tested qualitatively on the 42 trajectories, and this led to the conclusion that it is able to reason in a reliable manner about different types of situations, including in complex scenarios where the behaviour of the vehicle is inconsistent.

\section{B. Quantitative evaluation}

1) Evaluation procedure: Conventionally the performance of a system that estimates a driver's manoeuvre intention is evaluated by looking at how early and/or frequently it is able to make correct predictions. A prediction is considered correct if the true manoeuvre is found to be much more probable than the others, incorrect otherwise. This approach is not appropriate in our case because we evaluate our system in situations where it is not reasonable to expect such correct predictions. For example in the situation 2 b (Fig. 3) it is not possible for the system (or for a human) to estimate the driver's intention with high certainty from the clues provided by the map and the vehicle's behaviour. Therefore we need to look more closely at the uncertainty of the prediction in the evaluation procedure, which should reflect that the output of the system is reasonable in this situation. 


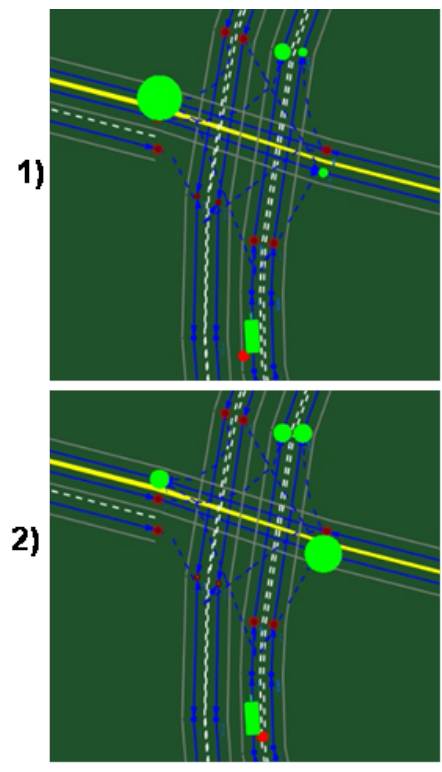

a)
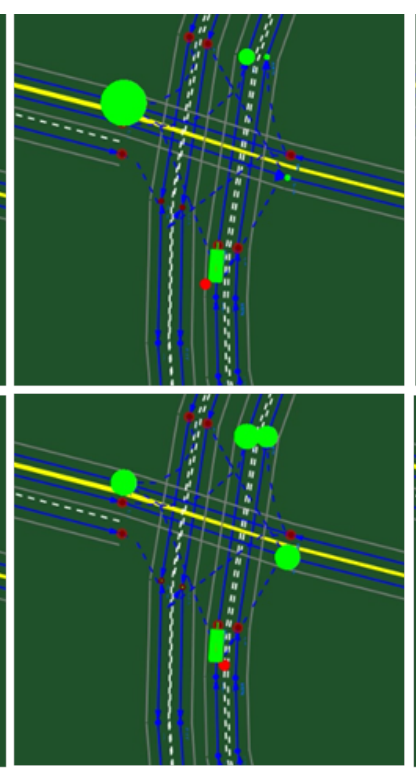

b)

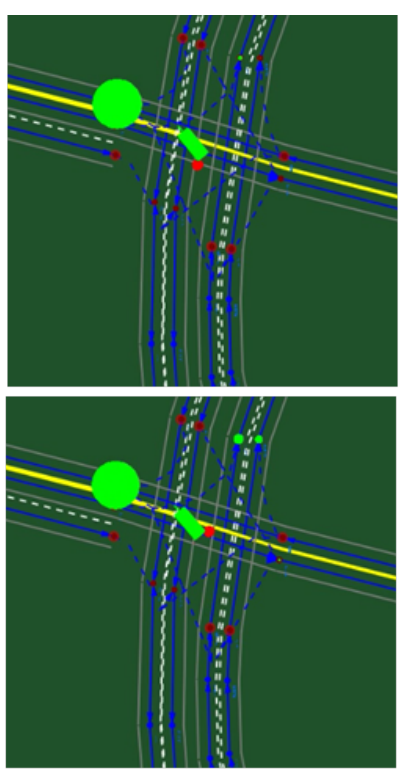

c)

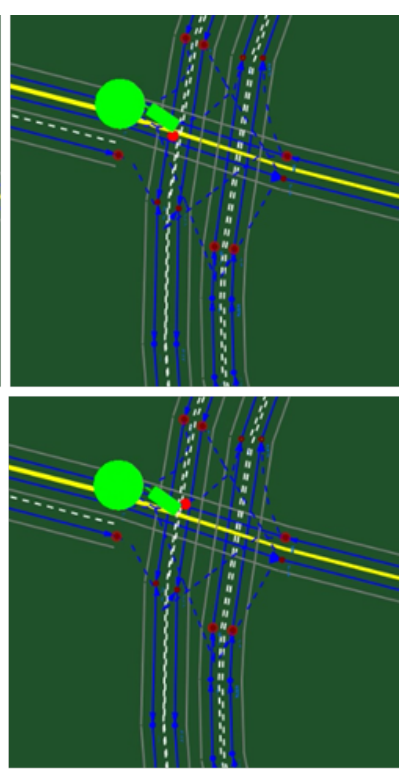

d)

Figure 3. Output of the system for a vehicle (green rectangle) approaching and traversing a road intersection ( $a \rightarrow b \rightarrow c \rightarrow d$ ) for a consistent behaviour scenario (row 1) and an inconsistent behaviour scenario (row 2). The pink circles displayed at the rear of the vehicle represent the turn signal that is on. The green circles located on the exit lanes show the probabilistic output of the system: the surface of each circle is proportional to the probability that the driver intends to reach that exit lane.

We define $m_{A} \in\left\{m_{i}\right\}_{1}^{N_{M}}$ as the most probable manoeuvre according to the system at time t, $m_{B} \in\left\{m_{i}\right\}_{1}^{N_{M}}$ as the second most probable manoeuvre according to the system at time t, and $m_{C} \in\left\{m_{i}\right\}_{1}^{N_{M}}$ as the true manoeuvre (i.e. the manoeuvre that is eventually performed by the driver). We consider three possible labels for a prediction at time t:

- Correct: $P\left(m_{A}\right)-P\left(m_{B}\right)>0.2$ and $m_{A}=m_{C}$.

- Incorrect: $P\left(m_{A}\right)-P\left(m_{B}\right)>0.2$ and $m_{A} \neq m_{C}$.

- Undecidable: $P\left(m_{A}\right)-P\left(m_{B}\right) \leq 0.2$. Intuitively, undecidable predictions correspond to cases where the system is not able to interpret a vehicle's behaviour.

2) Results and interpretation: The evaluation was run on the 42 trajectories for each type of scenario (consistent / inconsistent). The graphs in Fig. 4 display the obtained results.

a) Consistent behaviour scenarios: there are no incorrect predictions, which is not surprising since the behaviour of the vehicles is not misleading. The occasional undecidable predictions originate from situations where a vehicle is temporarily driving in between two lanes (or paths) and the clue given by the turn signal (or the absence of it) is not discriminating enough to identify which exit the driver is aiming at.

b) Inconsistent behaviour scenarios: in these scenarios the turn signal information is misleading, which explains why the manoeuvres are identified correctly much later than in the consistent scenarios. The system's response can be broken into three phases:

- Phase 1 corresponds to the early approach phase, when the vehicle is still more than $10 \mathrm{~m}$ away from the entrance of the intersection. On average $55 \%$ of the predictions are incorrect and $45 \%$ are undecidable. The incorrect predictions correspond situations where there exists a consistent explanation for the presence of the turn signal. An example is the situation 2a of Fig. 3: the vehicle is still far away from the intersection, so the right turn signal is interpreted as an intention to change lanes. A human would make the same interpretation, but it turns out to be an incorrect prediction. Another example is a vehicle on the left lane with the left turn signal on that eventually goes straight. The undecidable predictions correspond to cases where the system cannot find a consistent explanation for the vehicle's behaviour. As a result the uncertainty on the manoeuvre intention is high.

- Phase 2 corresponds to the end of the approach phase, when the vehicle is less than $10 \mathrm{~m}$ away from the entrance of the intersection. The trend in this phase is for the percentage of undecidable predictions to rise quickly while the percentage of incorrect predictions drops considerably. This inversion (incorrect predictions being replaced by undecidable predictions) is explained by the fact that as vehicles get closer to the intersection it is less and less likely that the drivers intend to change lanes. This is illustrated by situations $2 \mathrm{a}$ and $2 \mathrm{~b}$ in Fig. 3: the system's prediction is incorrect in 2a (Phase 1), and undecidable in $2 \mathrm{~b}$ (Phase 2) because there no longer exists a consistent explanation for the vehicle's behaviour.

- Phase 3 corresponds to the execution of the manoeuvre in the intersection. New clues appear (path that a vehicle follows in the intersection) and compete with the misleading turn signal information. As the vehicles get closer to an exit lane, a larger number of manoeuvres become unfeasible and therefore the system is able to identify the driver's intention despite the misleading turn signal 

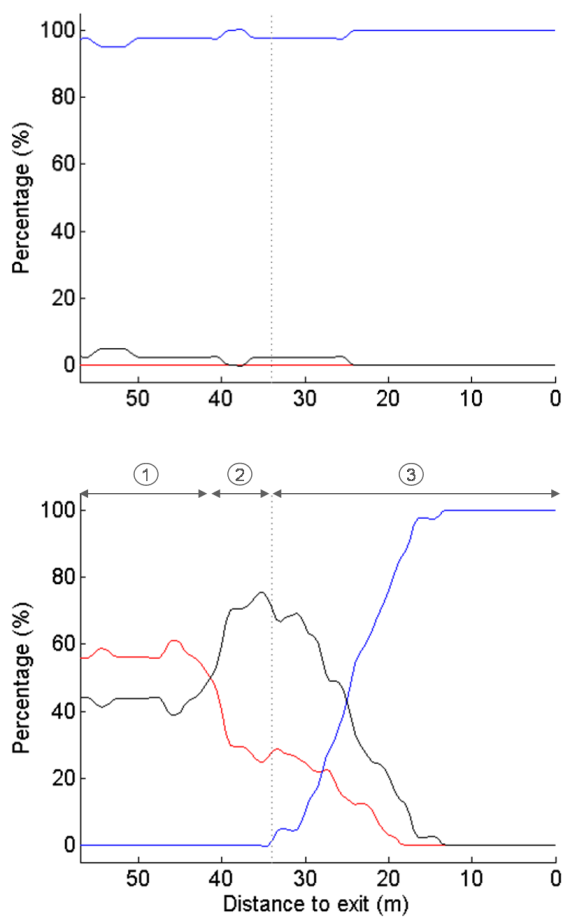

Figure 4. Results obtained on 42 trajectories with consistent behaviours (top) and on the same 42 trajectories with inconsistent behaviours (bottom). The graphs display the percentage of correct (blue), incorrect (red) and undecidable (black) predictions as a function of the distance to the exit of the intersection that the driver eventually reaches. The vertical dashed line represents the entrance of the intersection (i.e. the position of the stop line). In the bottom graph the progress of the vehicle is broken into three phases for ease of interpretation in Sec. IV-B.

information. We observe a steady rise of the percentage of correct predictions until it reaches $100 \%$ approximately $10 \mathrm{~m}$ away from the exit of the intersection.

Analysing the uncertainty of the system's output allowed us to to demonstrate the ability of our system to 1) make correct predictions in cases where there are enough clues to identify the manoeuvre intention and 2) keep the uncertainty on the prediction high when clues are lacking or inconsistent.

\section{CONCLUSIONS}

Situation assessment at road intersections is a key challenge for safety related driving assistance functions and autonomous driving. In this paper, the problem of estimating a driver's intended manoeuvre at a road intersection was addressed. The focus was on two aspects: 1) extracting and using the geometrical and topological characteristics of the intersection from a digital map, 2) handling uncertain evidence over lane assignment. A Bayesian network with uncertain evidence was proposed that combines observations on a vehicle's behaviour with information about the layout of the intersection in order to estimate of the manoeuvre that a driver intends to perform.

The system was tested on trajectories recorded from real traffic, in situations where the driver's behaviour was consistent and situations where it was inconsistent. We defined an evaluation method that takes into account that the performance of the system is not only about its ability to make correct predictions but also about its ability to identify ambiguous situations where the behaviour of a vehicle cannot be reliably interpreted. The results demonstrated the ability of the system to make reliable interpretations of the situations presented to it.

The model formulated in this paper does not take into account the kinematic behaviour of a vehicle to infer the driver's intended manoeuvre. Current work is extending the proposed approach and exploring the influence of traffic rules and vehicle interactions on a vehicle's behaviour at a road intersection.

\section{ACKNOWLEDGEMENTS}

This work is the result of a collaboration with the Stanford Artificial Intelligence Lab. The authors would like to thank Prof. S. Thrun for accepting the first author as a visiting researcher in his group, as well as Dr. S. Kammel and M. Sokolsky for their support in integrating this work into the Stanford Driving Group framework.

\section{REFERENCES}

[1] "Sunflower: a comparative study of the development of road safety in Sweden, the United Kingdom and the Netherlands," http://www.swov.nl/rapport/sunflower/sunflower.pdf.

[2] A. Lie and C. Tingvall, "Government Status Report, Sweden," Swedish Road Administration, 2001, http://wwwnrd.nhtsa.dot.gov/pdf/esv/esv21/09-0595.pdf.

[3] TRACE project, http://www.trace-project.org/.

[4] U.S. Department of Transportation / Federal Highway Administration Intersection Safety, http://safety.fhwa.dot.gov/intersection/.

[5] M. R. Endsley, "Toward a theory of situation awareness in dynamic systems: situation awareness," Human factors, vol. 37, no. 1, pp. 32 64, 1995.

[6] DARPA Urban Challenge, http://www.darpa.mil/grandchallenge.

[7] M. Montemerlo, J. Becker, S. Bhat, H. Dahlkamp, D. Dolgov, S. Ettinger, D. Haehnel, T. Hilden, G. Hoffmann, B. Huhnke, D. Johnston, S. Klumpp, D. Langer, A. Levandowski, J. Levinson, J. Marcil, D. Orenstein, J. Paefgen, I. Penny, A. Petrovskaya, M. Pflueger, G. Stanek, D. Stavens, A. Vogt, and S. Thrun, "Junior: the Stanford entry in the Urban Challenge," Journal of Field Robotics, vol. 25, no. 9, pp. 569597, 2008

[8] S. Atev, G. Miller, and N. Papanikolopoulos, "Clustering of vehicle trajectories," IEEE Transactions on Intelligent Transportation Systems, vol. 11 , no. 3, pp. 647-657, 2010.

[9] E. Kaefer, C. Hermes, C. Woehler, H. Ritter, and F. Kummert, "Recognition of situation classes at road intersections," in Proc. IEEE International Conference on Robotics and Automation, 2010, pp. 39603965.

[10] T. Hulnhagen, I. Dengler, A. Tamke, T. Dang, and G. Breuel, "Maneuver recognition using probabilistic finite-state machines and fuzzy logic," in Proc. IEEE Intelligent Vehicles Symposium, 2010, pp. 65-70.

[11] A. Kurt, J. L. Yester, Y. Mochizuki, and Ümit Özgüner, "Hybrid-state driver/vehicle modelling, estimation and prediction," in Proc. IEEE Intelligent Transportation Systems Conference, 2010, pp. 806-811.

[12] C. Tay, "Analysis of dynamic scenes: application to driving assistance," Ph.D. dissertation, Institut National Polytechnique de Grenoble, France, 2009.

[13] PReVENT project - INTERSAFE subproject, "Final report (d40.75)," February 2007.

[14] S. Lefèvre, C. Laugier, J. Ibañez-Guzmán, and P. Bessière, "Modelling dynamic scenes at unsignalised road intersections," INRIA Research Report 7604, April 2011.

[15] R. Schubert and G. Wanielik, "A unified Bayesian approach for tracking and situation assessment," in Proc. IEEE Intelligent Vehicles Symposium, 2010, pp. 738-745.

[16] J. Pearl, Probabilistic reasoning in intelligent systems: networks of plausible inference. Morgan Kaufmann, 1988. 